\title{
Simultaneous Imaging of Pseudomonas fluorescens WCS365 Populations Expressing Three Different Autofluorescent Proteins in the Rhizosphere: New Perspectives for Studying Microbial Communities
}

\author{
Guido V. Bloemberg, André H. M. Wijfjes, Gerda E. M. Lamers, Nico Stuurman, \\ and Ben J. J. Lugtenberg \\ Leiden University, Institute of Molecular Plant Sciences, Wassenaarseweg 64, Leiden 2333 AL, \\ The Netherlands \\ Accepted 20 July 2000.
}

To visualize simultaneously different populations of pseudomonads in the rhizosphere at the single cell level in a noninvasive way, a set of four rhizosphere-stable plasmids was constructed expressing three different derivatives of the green fluorescent protein (GFP), namely enhanced cyan (ECFP), enhanced green (EGFP), enhanced yellow (EYFP), and the recently published red fluorescent protein (RFP; DsRed). Upon tomato seedling inoculation with Pseudomonas fluorescens WCS365 populations, each expressing a different autofluorescent protein followed by plant growth for 5 days, the rhizosphere was inspected using confocal laser scanning microscopy. We were able to visualize simultaneously and clearly distinguish from each other up to three different bacterial populations. Microcolonies consisting of mixed populations were frequently observed at the base of the root system, whereas microcolonies further toward the root tip predominantly consisted of a single population, suggesting a dynamic behavior of microcolonies over time. Since the cloning vector pME6010 has a broad host range for gram-negative bacteria, the constructed plasmids can be used for many purposes. In particular, they will be of great value for the analysis of microbial communities, for example in processes such as biocontrol, biofertilization, biostimulation, competition for niches, colonization, and biofilm formation.

Additional keywords: fluorescence, triple color imaging.

Biological control of soilborne pathogens by Pseudomonas fluorescens is usually based on (i) the production of an antifungal factor (AFF) by the bacterium and (ii) an efficient delivery of this AFF along the root system by root colonization (Lugtenberg et al. 1991, 2000; Thomashow and Weller 1995). However, the efficacy of biocontrol bacteria requires further improvement. Therefore, fundamental knowledge about spatiotemporal interactions between the bacterium, the plant, the

Corresponding author: G. V. Bloemberg; Telephone: +31-71-5275056; Fax: +31-71-5275088; E-mail: bloemberg@ rulbim.leidenuniv.nl phytopathogenic fungus, and the endogenous microbial population are required. In order to visualize and understand Pseudomonas root colonization and interactions with other bacteria and fungi in the rhizosphere, we expressed multiple autofluorescent proteins (AFPs).

Green fluorescent protein (GFP), isolated from the jellyfish Aequorea victoria, has been the most revolutionary reporter in biology since its application as a marker was published by Chalfie et al. (1994). The major advantage of GFP as a reporter is its noninvasive analysis without the need for exogenous substrates or energy. The GFP is a very suitable marker for studying bacterial behavior at the single cell level in the rhizosphere, such as Pseudomonas root colonization (Bloemberg et al. 1997; Normander et al. 1999; Ramos et al. 2000; Tombolini et al. 1997, 1999) and rhizobial nodulation (Gage et al. 1996; $\mathrm{Xi}$ et al. 1999). Recently, color variants of the GFP, e.g., enhanced cyan (ECFP), enhanced green (EGFP), and enhanced yellow (EYFP), with shifted excitation and emission maxima have been developed and used for dual color imaging (Ellenberg et al. 1999; Matus 1999; Tsien 1998; Yang et al. 1998). Most recently, the red fluorescent protein (RFP; DsRed) (Matz et al. 1999) isolated from Discosoma spp. has broadened the range of AFPs, creating the opportunity for triple color imaging.

We report the construction of four rhizosphere-stable plasmids for constitutive expression of ecfp, egfp, eyfp, and $r f p$ (DsRed), respectively, in P. fluorescens, rhizobia, and other gram-negative bacteria. Using confocal laser scanning microscopy, we showed that all four plasmids are highly suitable reporter vectors for visualization of bacteria at the single cell level in the rhizosphere. Here, we report, for what we believe is the first time, dual and even triple imaging of mixed Pseudomonas populations in the rhizosphere, each expressing a different AFP.

\section{RESULTS}

Construction of rhizosphere-stable plasmids for the expression of AFP in P. fluorescens.

A set of four rhizosphere-stable plasmids was constructed to express the egfp, ecfp, eyfp, and $r f p$ genes under control of the 
lac promoter (Fig. 1), which resulted in plasmids pMP2444, pMP4516, pMP4518, and pMP4661, respectively. Since pBBR1-based plasmids appeared not to be stable in $P$. fluorescens during subsequent subculturing in medium without antibiotic pressure (data not shown), they were fused to the rhizosphere-stable cloning vector pME6010, which is based on the pVS1 replicon (Heeb et al. 2000). This resulted in plasmids pMP4655, pMP4641, pMP4658, and pMP4662, respectively (Fig. 1). The latter plasmids were transformed into the efficient root-colonizing strain P. fluorescens WCS365 and tested for stability in the rhizosphere. After testing several hundreds of bacteria for each construct reisolated from the root tip of tomato plants 7 days after seedling inoculation and subsequent plant growth in a gnotobiotic quartz sand system, no loss of the plasmids was observed. The constructs were also positively tested for stability in the rhizosphere bacterium Rhizobium spp. (data not shown) (Stuurman et al. 2000); no loss of the plasmid was observed after reisolation from the rhizosphere. Apparently, pME6010 tolerates insertions of pBBR1 in both orientations, without loss of plasmid stability. Expression of the AFP genes in Escherichia coli as well as in P. fluorescens WCS365 was confirmed by epifluorescence microscopic analysis. However, expression of DsRed could hardly be detected after growth of $E$. coli at $37^{\circ} \mathrm{C}$ and $P$. fluorescens $\mathrm{WCS} 365$ at $28^{\circ} \mathrm{C}$. This was probably due to instability at these temperatures, since expression of DsRed in P. fluorescens could easily be detected on tomato root surfaces of plants grown at $21^{\circ} \mathrm{C}$. Expression of the different AFP genes had no significant effect on the growth rate of $P$. fluorescens WCS365 when the strains were grown in liquid King's medium B (KB) but resulted in a slightly longer lag phase (Fig. 2) in comparison to the wild-type strain WCS365.

\section{Quantification of AFPs expressed in E. coli and P. fluorescens.}

Quantification of AFP expression in E. coli and P. fluorescens (Fig. 3) was performed to (i) compare the relative intensities of the different AFPs, (ii) compare expression of the AFPs in E. coli and P. fluorescens, and (iii) analyze the overlap in excitation and emission of the strains expressing ecfp, egfp, or eyfp. Expression of $r f p$ (encoding DsRed) in E. coli

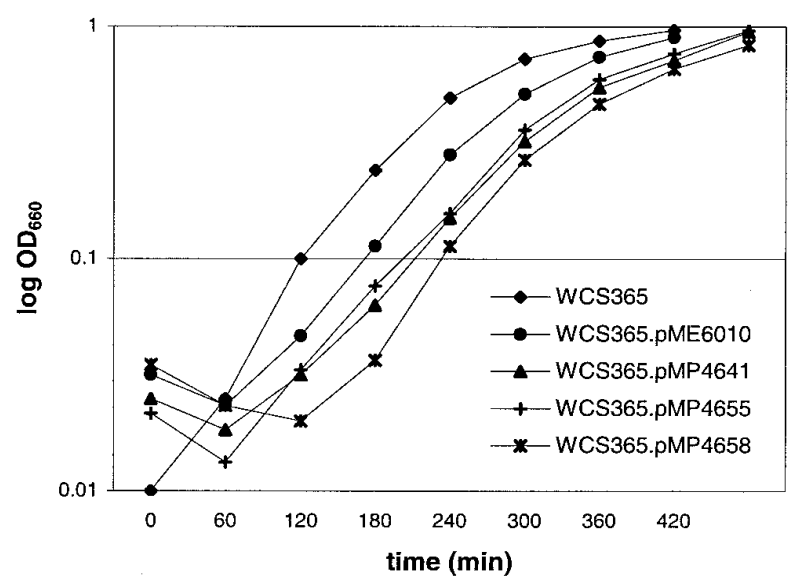

Fig. 2. Growth of Pseudomonas strains expressing afp genes. Overnight cultures of $P$. fluorescens WCS365 harboring pME6010, pMP4641 (ecfp), pMP4655 (egfp), and pMP4658 (eyfp), respectively, were diluted to an optical density at $660 \mathrm{~nm}$ of 0.01 to 0.5 in fresh King's medium B. Optical density of the cultures was measured during growth at $28^{\circ} \mathrm{C}$ under vigorous shaking. The presented data are mean values of three replicate experiments.

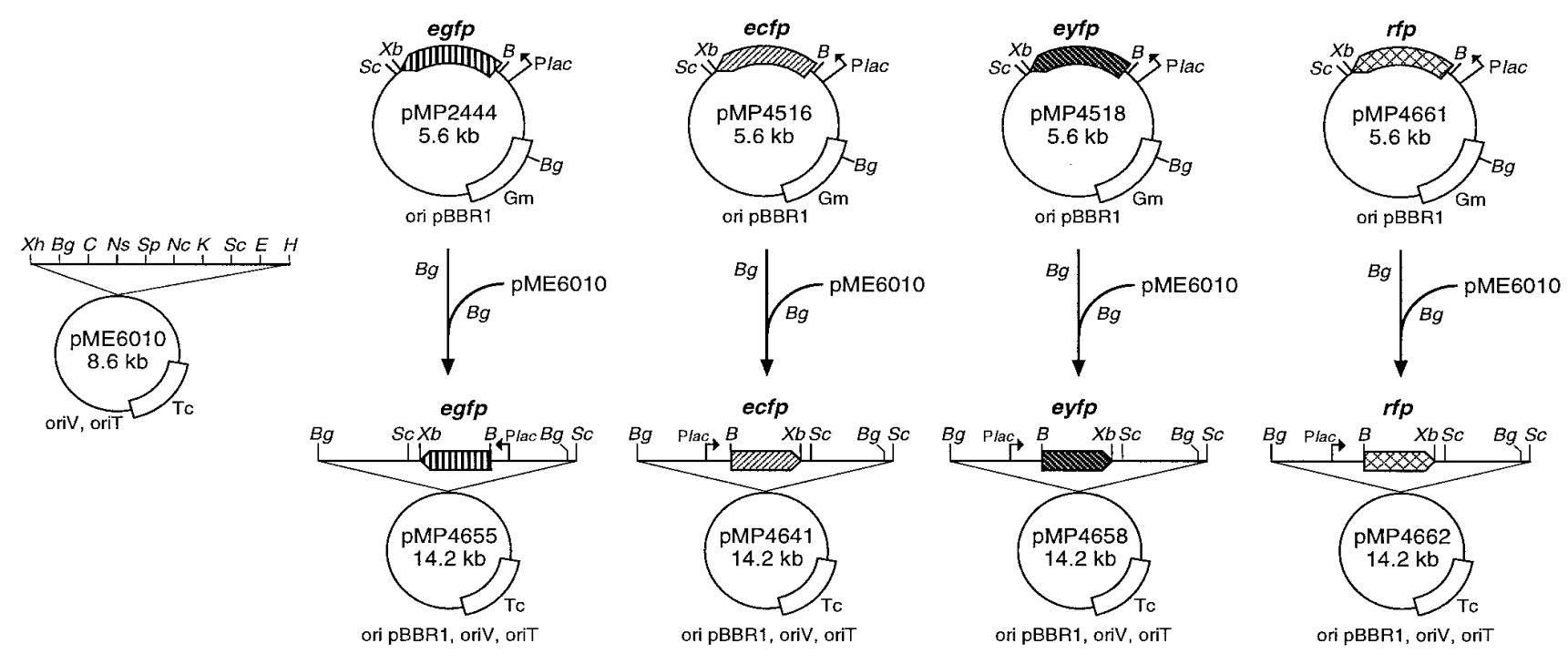

Fig. 1. Construction of plasmids to express autofluorescent proteins in gram-negative bacteria. Plasmid pME6010 (Heeb et al. 2000) was used as a cloning vector to construct rhizosphere-stable reporter plasmids expressing egfp, ecfp, eyfp, and $r f p$, respectively, under the control of the lac promoter. Plasmids pMP2444, pMP4516, pMP4518, and pMP4661, all containing an origin of replication of the pBBR1 class, were fused with pME6010 by restriction with $B g l I I$ followed by ligation, resulting in pMP4655, pMP4641, pMP4658, and pMP4662, respectively. Orientations of the autofluorescent protein genes are indicated. Abbreviations: $\mathrm{B}=B a m \mathrm{HI}, \mathrm{Xh}=X h o \mathrm{I}, \mathrm{Bg}=B g l \mathrm{II}, \mathrm{C}=C l a \mathrm{I}, \mathrm{Ns}=N s i \mathrm{I}, \mathrm{Sp}=S p h \mathrm{I}, \mathrm{Nc}=N c o \mathrm{I}, \mathrm{K}=K p n \mathrm{I}, \mathrm{E}=E c o \mathrm{RI}, \mathrm{H}=$ HindIII, $\mathrm{Sc}=S a c \mathrm{I}, \mathrm{Xb}=X b a \mathrm{I}, \mathrm{Tc}=$ tetracycline, $\mathrm{Gm}=$ gentamicin, $\mathrm{Plac}=$ lac promoter, $e g f p=$ enhanced green fluorescent protein, e $c f p=$ enhanced cyan fluorescent protein, eyfp $=$ enhanced yellow fluorescent protein, and $r f p=$ DsRED protein. 
and P. fluorescens was not included, since we could not observe expression by epifluorescence microscopy in E. coli and $P$. fluorescens. Under the filter conditions of the fluorometer, strains expressing egfp show the highest relative fluorescence,
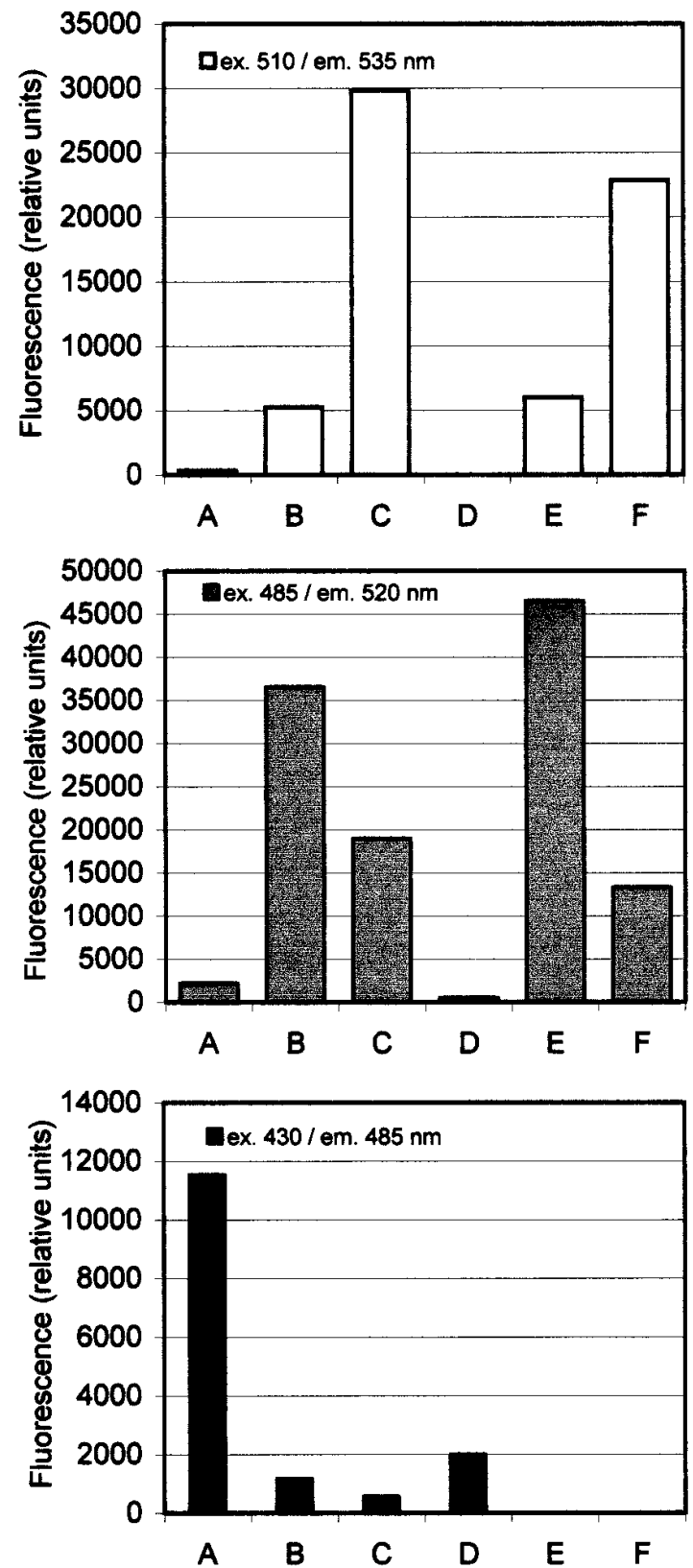

Fig. 3. Quantification of autofluorescent proteins in Escherichia coli and Pseudomonas fluorescens. Overnight cultures of $E$. coli DH5 $\alpha$ and $P$. fluorescens WCS365 harboring plasmids pMP4641 (ecfp), pMP4655 (egfp), or pMP4658 (eyfp), respectively, were diluted into fresh Luria-Bertani medium or King's medium B to an optical density at $660 \mathrm{~nm}$ of 0.6. Fluorescence of the diluted cultures was quantified in a 96-well titer plate containing aliquots of 200- $\mu \mathrm{l}$ cultures using a plate reader fluorometer with excitation (ex) and emission (em) filters for analysis of enhanced yellow fluorescent protein (EYFP; ex $510 \mathrm{~nm}$ and em $535 \mathrm{~nm}$ ), enhanced green fluorescent protein (EGFP; ex $485 \mathrm{~nm}$ and em $520 \mathrm{~nm}$ ), and enhanced cyan fluorescent protein (ECFP; ex $430 \mathrm{~nm}$ and em $485 \mathrm{~nm}$ ), respectively. Depicted in this figure are the emission values of strains $E$. coli DH5 $\alpha$ harboring A, pMP4641; B, pMP4655; or C, pMP4658 and P. fluorescens WCS365 harboring D, pMP4641; E, pMP4655; or F, pMP4658. The presented data were corrected for autofluorescence. followed by strains expressing eyfp and ecfp, respectively (Fig. 3). Expression of the afp genes could easily be detected in all strains, with relatively small differences between $E$. coli and $P$. fluorescens, except ecfp expression was much lower (but still clearly detectable) in P. fluorescens than in E. coli. Figure 3 shows that excitation and emission for EGFP and EYFP clearly overlap as can be expected from their excitation and emission spectra (Tsien 1998). However, negligible overlap of the emission and excitation data between ECFP and EGFP as well as between ECFP and EYFP was observed.

\section{Confocal laser scanning microscopy analysis of tomato root colonization by $P$. fluorescens expressing various AFPs.}

In order to visualize $P$. fluorescens bacteria colonizing tomato roots, 2-day-old germinated tomato seedlings, with roots of approximately $1 \mathrm{~cm}$ in length, were inoculated with $P$. fluorescens WCS365 derivatives expressing ECFP, EGFP, EYFP, or DsRed. After inoculation, the seedlings were grown in a gnotobiotic quartz sand system for 5 days and the root systems with an average length of $7 \mathrm{~cm}$ subsequently examined for the presence of bacterial cells using confocal laser scanning microscopy. Microcolonies consisting of mixed populations were predominantly observed in the upper half of the root system, whereas colonies in the lower half tended to consist of predominantly one population. However, mixed microcolonies could still be observed in the lower part. Figure 4 shows images of $P$. fluorescens microcolonies on the tomato root surface present in the region located between 3 and $4 \mathrm{~cm}$ below the root base. Confocal microscope images of tomato plants inoculated with single $P$. fluorescens populations show that ecfp (Fig. 4A), egfp (Fig. 4B), eyfp (Fig. 4C), and DsRed (Fig. 4D) are expressed in the rhizosphere and are all highly suitable markers for visualization of bacteria at the single cell level in the rhizosphere. The latter observation was remarkable, since expression of $r f p$ in $P$. fluorescens was not observed by epifluorescence microscopy when cells were grown at $28^{\circ} \mathrm{C}$. Subsequently, tomato seedlings were inoculated with mixed cultures of two differently labeled $P$. fluorescens WCS365 derivatives. Pseudomonas cells expressing ecfp and egfp could clearly be distinguished from each other in microcolonies (Fig. 5A). The same applies for Pseudomonas cells expressing ecfp and eyfp (Fig. 5B and C). Since the emission spectrum of DsRed does not overlap with that of the other AFPs, tomato seedlings were inoculated with a mixture of three $P$. fluorescens derivatives expressing ecfp, egfp, and $r f p$, respectively. Triple imaging of root surfaces after growth in the gnotobiotic sand system resulted in visualization of microcolonies consisting of all three populations (Fig. 5D). Bacteria expressing the three different AFPs could easily be distinguished from each other.

\section{DISCUSSION}

In order to study root colonization and interactions of (biocontrol) pseudomonads in the rhizosphere, a set of reporter vectors was constructed for constitutive expression of ecfp, egfp, eyfp, and $r f p$, respectively, under control of the lac promoter (Fig. 1). In P. fluorescens, the resulting plasmids were shown to be stable during growth in the rhizosphere (without antibiotic pressure). Since the plasmids are based on 
cloning vector pME6010 (Heeb et al. 2000), which contains a pVS1 replicon, the constructed plasmids are presumably stable in many other gram-negative bacteria and highly suitable to visualize bacteria in environments where no antibiotic pressure can be applied. We show here that expression of AFPs under control of the lac promoter does not influence the growth rate of $P$. fluorescens in $\mathrm{KB}$, although the lag time was extended (Fig. 2). Expression of AFPs can easily be quantified using a plate reader fluorometer, which provides opportunities for gene expression studies.

Due to (i) highly advanced confocal laser microscopy equipment that allows sequential scanning and detection of emitted light at freely selectable wavelengths and (ii) the recent availability of the DsRed protein (Matz et al. 1999), we
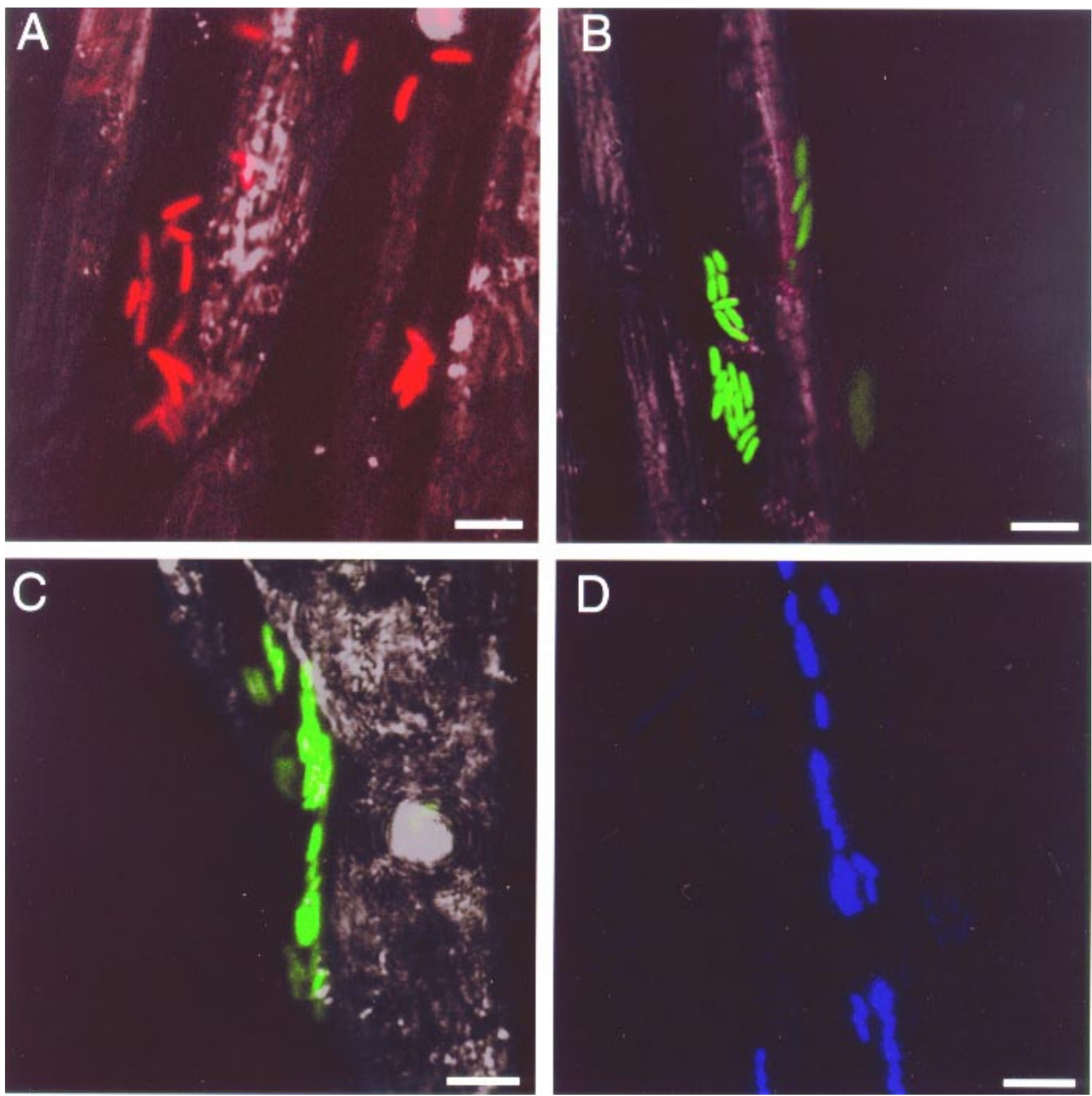

Fig. 4. Confocal laser scanning microscopical analysis of tomato root colonization by Pseudomonas fluorescens WCS365 expressing autofluorescent proteins. Two-day-old germinated tomato seedlings were inoculated with P. fluorescens WCS365 harboring reporter plasmids expressing various afp genes. After inoculation, plants were grown in a gnotobiotic quartz sand system. After 5 days of growth in the gnotobiotic system, roots were examined for the presence of fluorescent bacteria by confocal laser scanning microscopy (details in Materials and Methods). Tomato root surfaces colonized by $P$. fluorescens WCS365 harboring A, pMP4641 (ecfp); B, pMP4655 (egfp); C, pMP4658 (eyfp); or D, pMP4662 (rfp). Enhanced cyan fluorescent protein (ECFP)-expressing bacteria appear red, enhance green fluorescent protein (EGFP)- and enhanced yellow fluorescent protein (EYFP)-expressing bacteria green, and red fluorescent protein (DsRed)-expressing bacteria blue. The size bar represents $5 \mu \mathrm{m}$ in all panels. 
have been able to tag Pseudomonas bacteria with four different AFPs and to visualize them in the rhizosphere (Fig. 4). It was shown that Pseudomonas populations expressing ecfp, $r f p$, and egfp or eyfp were clearly distinguishable from each other in the rhizosphere (Fig. 5). We succeeded, for what we believe is the first time, in visualizing and clearly distinguishing three different Pseudomonas populations simultaneously in the rhizosphere in a noninvasive way (Fig. 5D). Visualization of mixed $P$. fluorescens populations showed that mixed microcolonies can easily be detected in the upper part
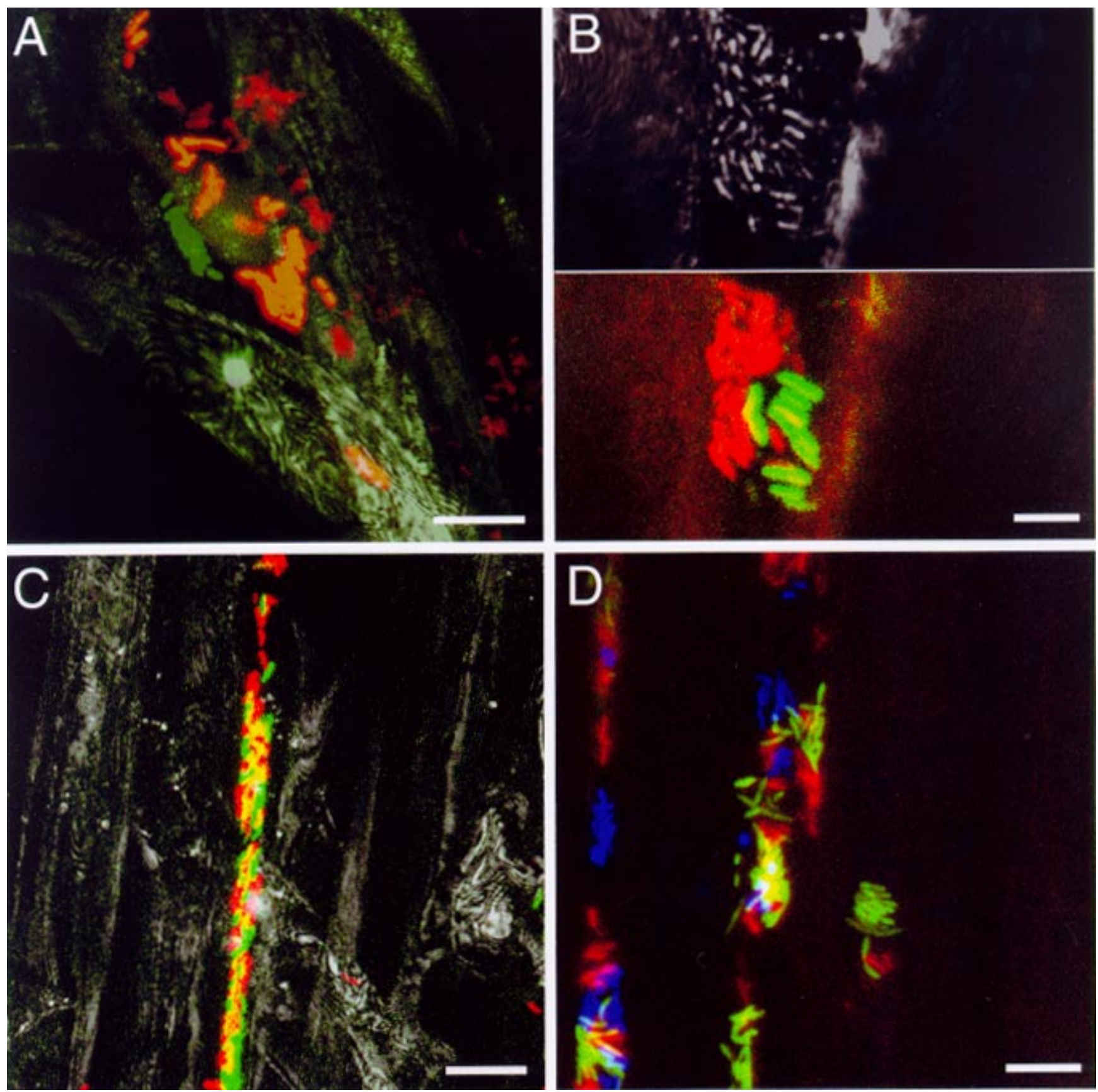

Fig. 5. Confocal laser scanning microscopical analysis of tomato root colonization by Pseudomonas fluorescens WCS365 expressing different autofluorescent proteins. A, B, and $\mathbf{C}$, Parts of the lower half of tomato roots, the seedlings of which were inoculated with mixtures of two $P$. fluorescens WCS365 derivatives expressing different $a f p$ genes (details on growth in Fig. 4 caption): A, pMP4641 (ecfp) and pMP4655 (egfp); B and C, pMP4641(ecfp) and pMP4658 (eyfp). B, The upper part shows an image obtained by measuring the light reflected by surfaces present in the object, whereas the lower part shows the same area with only the enhanced cyan fluorescent protein (ECFP)-specific fluorescence in red and the enhanced yellow fluorescent protein (EYFP)-specific fluorescence in green. D, Part of the root surface of a tomato plant, the seedling of which had been inoculated with a mixture of three $P$. fluorescens WCS365 derivatives harboring pMP4641 (ecfp), pMP4655 (egfp), and pMP4662 (rfp), respectively. In the panels, enhanced cyan fluorescent protein (ECFP)-expressing bacteria appear red, enhanced green fluorescent protein (EGFP) and enhanced yellow fluorescent protein (EYFP) expressing bacteria appear green, and red fluorescent protein (DsRed)-expressing bacteria appear blue. White represents the reflected light. $\mathbf{C}$ and $\mathbf{D}$, Yellow color is caused by overlap of red and green colors. The size bars represents $\mathbf{A}$ and $\mathbf{B}, 5 \mu \mathrm{m}$; and $\mathbf{C}$ and $\mathbf{D}, 10 \mu \mathrm{m}$. 
of the root system. In the lower part of the root system, colonies completely consisted of one population, to which sometimes several bacteria (visible as single cells) from the other population are attached. These results suggest the following sequence of events. (i) Microcolonies are started by one bacterium that will divide over time, resulting in the formation of the microcolony. (ii) Other bacteria can reach the same site at a later time point and can become part of the same microcolony. (iii) The site to be colonized is most likely a site of exudation.

Currently, many studies of bacteria in natural environments show that they live in complex communities, predominantly in biofilms, where they interact with other species. The novelty of the results presented in this paper provides many new perspectives for analyzing the formation and function of microbial populations and communities.

\section{MATERIALS AND METHODS}

\section{Bacterial strains and growth conditions.}

P. fluorescens strain WCS365 (Geels and Schippers 1983; Simons et al. 1996) was routinely cultured in KB (King et al. $1954)$ at $28^{\circ} \mathrm{C}$. When appropriate, tetracycline was added to the culture medium to a final concentration of $80 \mu \mathrm{g} / \mathrm{ml}$. E. coli strain DH5 $\alpha$ was routinely cultured in Luria-Bertani medium (LB) (Sambrook et al. 1989) and, when appropriate, supplemented with tetracycline (final concentration of $20 \mu \mathrm{g} / \mathrm{ml}$ ) or gentamicin (final concentration of $10 \mu \mathrm{g} / \mathrm{ml}$ ).

\section{Construction of plasmids, plasmid stability, and $\boldsymbol{a f p}$ expression.}

Initially, egfp, ecfp, and eyfp (Clontech, Palo Alto, CA, U.S.A.) were cloned into the broad-host-range vector pBBR1MCS-5 (Kovach et al. 1995), resulting in plasmids pMP2444, pMP4516, and pMP4518, respectively (Stuurman et al. 2000). Introduction of these plasmids into P. fluorescens strain WCS365 resulted in clear expression of the various AFP genes (data not shown). To obtain rhizospherestable plasmids expressing afps, plasmids pMP2444, pMP4516, and pMP4518 were restricted with $B g l$ II and fused by ligation with pME6010 (Heeb et al. 2000), resulting in plasmids pMP4655, pMP4641, and pMP4658, respectively (Fig. 1). In a later stage, the very recently marketed pDsRED vector (Clontech) was used to clone the DsRed gene as a BamHI-XbaI fragment into cloning vector pBBR1MCS-5, resulting in plasmid pMP4661. Fusion of pMP4661 with pME6010 using the mutual BglII site resulted in plasmid pMP4662 (Fig. 1). After transforming these plasmids to $P$. fluorescens WCS365 by electroporation, stability of the plasmids in the rhizosphere (without the presence of antibiotic pressure) was determined after inoculating tomato seedlings, as described below, with the WCS365 derivatives and growth of the seedlings in a gnotobiotic quartz sand system. After 7 days, bacteria were isolated from the root tip as described before (Simons et al. 1996) and plated on KB agar plates without antibiotics. From each WCS365 derivative, 200 colonies were subsequently tested for the presence of the plasmid on KB supplemented with tetracycline. Expression of egfp, ecfp, and eyfp in E. coli and P. fluorescens was quantified using a
HTS7000 Bio Assay Reader (Perkin \& Elmer Life Sciences, Oosterhout, The Netherlands). Overnight cultures were diluted in fresh LB or KB to an optical density at $660 \mathrm{~nm}$ of 0.6 using a LKB Biochrom Nova Spectrometer (Amersham Pharmacia Biotech, Roosendaal, The Netherlands). Fluorescence of the diluted cultures was quantified using a white, 96-well titer plate containing 200- $\mu$ l culture aliquots. Fluorescence of the cultures was determined with excitation filters having maxima at $430( \pm 35 \mathrm{~nm}), 485( \pm 20 \mathrm{~nm})$, and $510 \mathrm{~nm}( \pm 10 \mathrm{~nm})$ and emission filters having maxima at $485( \pm 20 \mathrm{~nm}), 520( \pm 10 \mathrm{~nm})$, and $535 \mathrm{~nm}( \pm 25 \mathrm{~nm})$ for quantification of ECFP, EGFP, and EYFP, respectively.

\section{Growth of tomato seedlings in a gnotobiotic test system.}

Tomato plants were grown in a gnotobiotic sand system as described previously by Simons et al. (1996). Tomato seeds (Lycopersicon esculentum Mill. cv. Carmello) were kindly provided by Novartis B.V., Enkhuizen, The Netherlands. To inoculate tomato seedlings overnight, bacterial cultures were diluted to $10^{7} \mathrm{CFU} / \mathrm{ml}$. Tomato seeds were sterilized, germinated, and inoculated, and seedlings were grown under conditions described previously (Simons et al. 1996).

\section{Microscopy.}

Bacteria harboring plasmids with AFP genes were examined using a Leica MZFLIII stereo microscope equipped with epifluorescence detection (Leica, Bensheim, Germany). Filter sets tailored to the specific chromophores were used (for ECFP, 440/21-nm excitation with 480/36-nm emission; for EGFP, 480/40-nm excitation with 510-nm long pass emission; for EYFP, 500/10-nm excitation with 518/16-nm emission; and for DsRed, 510/20-nm excitation with 560/40-nm emission).

Tomato roots colonized by $P$. fluorescens after 5 days of growth in the gnotobiotic sand system were washed in phosphate-buffered saline (PBS) to remove sand particles and mounted in PBS on an attached coverslip. Samples were examined with an inverted fluorescence microscope (DMIRBE; Leica) equipped with filter blocks with spectral properties matching those of ECFP (440/21-nm excitation with 480/36nm emission; XF114, Chroma, Brattleboro, VT, U.S.A.), or EGFP and EYFP (470/20-nm excitation with 515-nm long pass emission; I3, Leica), or DsRed (538/22-nm excitation with 590-nm emission long pass emission; N2.1, Leica), to which a Leica SP confocal scanhead was attached. Dual and triple color images were acquired by sequentially scanning with settings optimal for ECFP (excitation with the 457-nm argon laser line, emission detection between 470 and $490 \mathrm{~nm}$ ), followed by settings optimal for EGFP (excitation with the 488-nm argon laser line, detection of emitted light between 500 and $520 \mathrm{~nm}$ ) or EYFP (excitation with the 488-nm argon laser line, detection of emitted light between 530 and $550 \mathrm{~nm})$, and finally by settings optimal for DsRed (excitation with the 568-nm krypton laser line, detection of emitted light between 580 and $620 \mathrm{~nm}$ ). Reflected light images were obtained by detection of light at the wavelength used for excitation. Cross talk between the channels in this setup was always monitored and appeared to be negligible in all cases. The projections of the individual channels were merged in Photoshop 5.0 (Adobe, San Jose, CA, U.S.A.) to facilitate visualization. 


\section{ACKNOWLEDGMENTS}

We thank H. P. Spaink for very useful discussions and advice, S. Heeb and D. Haas for kindly providing the cloning vector pME6010, and P. Hock for his help in preparing figures. The purchase of microscopic equipment was supported by a grant of the Netherlands Organization of Scientific Research. The HTS7000 Bio Assay Reader was purchased with financial support of the "Gratema Stichting" and the "Leids Universiteits Fonds (LUF)."

\section{LITERATURE CITED}

Bloemberg, G. V., O’Toole, G. A., Lugtenberg, B. J. J., and Kolter, R. 1997. Green fluorescent protein as a marker for Pseudomonas spp. Appl. Environ. Microbiol. 63:4543-4551.

Chalfie, M., Tu, Y., Euskirchen, G., Ward, W. W., and Prasher, D. C. 1994. Green fluorescent protein as a marker for gene expression. Science 263:802-805.

Ellenberg, J., Lippincott, S. J., and Presley, J. F. 1999. Dual-colour imaging with GFP variants. Trends Cell Biol. 9:52-56.

Gage, D. J., Bobo, T., and Long, S. R. 1996. Use of green fluorescent protein to visualize the early events of symbiosis between Rhizobium meliloti and alfalfa (Medicago sativa). J. Bacteriol. 178:7159-7166.

Geels, F. P., and Schippers, B. 1983. Reduction of yield depressions in high frequency potato cropping soil after seed tuber treatments with antagonistic fluorescent Pseudomonas spp. Phytopathol. Z. 108:207214.

Heeb, S., Itoh, Y., Nishijyo, T., Schnider, U., Keel, C., Wade, J., Walsh, U., O'Gara, F., and Haas, D. 2000. Small, stable shuttle vectors based on the minimal pVS1 replicon for use in gram-negative, plantassociated bacteria. Mol. Plant-Microbe Interact. 13:232-237.

King, E. O., Ward, M. K., and Raney, D. E. 1954. Two simple media for the demonstration of pyocyanin and fluorescein. J. Lab. Clin. Med. 44:301-307.

Kovach, M. E., Elzer, P. H., Hill, D. S., Robertson, G. T., Farris, M. A., Roop, R. M., and Peterson, K. M. 1995. Four new derivatives of the broad-host-range cloning vector pBBR1MCS, carrying different antibiotic-resistance cassettes. Gene 166:175-176.

Lugtenberg, B. J. J., Dekkers, L. C., Bansraj, M., Bloemberg, G. V., Camacho, M., Chin-A-Woeng, T., van den Hondel, K., Kravchenko, L., Kuiper, I., Lagopodi, A. L., Mulders, I., Phoelich, C., Ram, A., Tikhonovich, I., Tuinman, S., Wijffelman, C., and Wijfjes, A. 2000. Pseudomonas genes and traits involved in tomato root colonization. Pages 324-330 in: Biology of Plant-Microbe Interactions, Vol. 2. P. J. G. M. de Wit, T. Bisseling, and W. J. Stiekema, eds. International Society for Molecular Plant-Microbe Interactions, St. Paul, MN, U.S.A.

Lugtenberg, B. J. J., de Weger, L. A., and Bennet, J. W. 1991. Microbial stimulation of plant growth and protection from disease. Curr. Opin. Biotechnol. 2:457-464.

Matus, A. 1999. GFP in motion CD-ROM-Introduction: GFP illuminates everything. Trends Cell Biol. 9:43.

Matz, M. M. V., Fradkov, A. F., Labas, Y. A., Savitsky, A. P., Zaraisky, A. G., Markelov, M. L., and Lukyanov, S. A. 1999. Fluorescent proteins from nonbioluminescent Anthozoa species. Nat. Biotechnol. 17:969-973.

Normander, B., Hendriksen, N. B., and Nybroe, O. 1999. Green fluorescent protein-marked Pseudomonas fluorescens: Localization, viability, and activity in the natural barley rhizosphere. Appl. Environ. Microbiol. 65:4646-4651.

Ramos, C., Molbak, L., and Molin, S. 2000. Bacterial activity in the rhizosphere analyzed at the single-cell level by monitoring ribosome content and synthesis rates. Appl. Environ. Microbiol. 66:801-809.

Sambrook, J., Fritsch, E. F., and Maniatis, T. 1989. Molecular Cloning. A Laboratory Manual. Cold Spring Harbor Laboratory Press, Cold Spring Harbor, NY, U.S.A.

Simons, M., van der Bij, A. J., Brand, I., de Weger, L. A., Wijffelman, C. A., and Lugtenberg, B. J. J. 1996. Gnotobiotic system for studying rhizosphere colonization by plant growth-promoting Pseudomonas bacteria. Mol. Plant-Microbe Interact. 9:600-607.

Stuurman, N., Pacios Bras, C., Schlaman, H. R. M., Wijfjes, A. H. M., Bloemberg, G. V., and Spaink, H. P. 2000. The use of green fluorescent protein color variants expressed on stable broad-host-range vectors to visualize rhizobia interacting with plants. Mol. Plant-Microbe Interact. 13:1163-1169.

Thomashow, L. S., and Weller, D. M. 1995. Current concepts in the use of introduced bacteria for biological disease control: Mechanisms and anti-fungal metabolites. Pages 187-235 in: Plant Microbe Interactions, Vol. 1. G. Stacey and N. Keen, eds. Chapman and Hall, New York.

Tombolini, R., Unge, A., Davey, M. E., de Bruijn, F. J., and Schleifer, K. H. 1997. Flow cytometric and microscopic analysis of GFP-tagged Pseudomonas fluorescens bacteria. FEMS Microbiol. Ecol. 22:17-28.

Tombolini, R., van der Gaag, D. J., Gerhardson, B., and Jansson, J. K. 1999. Colonization pattern of the biocontrol strain Pseudomonas chlororaphis MA32 on barley seeds visualized by using green fluorescent protein. Appl. Environ. Microbiol. 65:3674-3680.

Tsien, R. Y. 1998. The green fluorescent protein. Annu. Rev. Biochem. 67:509-544.

Xi, C., Lambrecht, M., Vanderleyden, J., and Michiels, J. 1999. Bifunctional $g f p$ - and gusA-containing mini-Tn5 transposon derivatives for combined gene expression and bacterial localization studies. J. Microbiol. Methods 35:85-92.

Yang, T. T., Sinai, P., Green, G., Kitts, P. A., Chen, Y. T., Lybarger, L., Chervenak, R., Patterson, G. H., Piston, D. W., and Kain, S. R. 1998 Improved fluorescence and dual color detection with enhanced blue and green variants of the green fluorescent protein. J. Biol. Chem. 273:8212-8216. 